\title{
A REMARK ON ORTHOGONALITY RELATIONS IN FINITE GROUPS
}

\author{
To RICHARD BRAUER on his 60 th birthday \\ KENZO IIZUKA and TADASI NAKAYAMA
}

On the basis of Prof. R. Brauer's fundamental work, certain orthogonality relations for characters of finite groups have recently been studied by Brauer himself, M. Osima, and one of the present writers; see lizuka [7] and the references there. In the present short note some general remarks on orthogonality relations, dealing with "blocks" and "sections" of general type, are given first. They are of elementary, and often formal, nature and their proofs are merely combinations of known arguments. So, no deep significance is claimed on them, in comparison with the above alluded results based on deeper arithmetico-group-theoretical considerations. However, applied to blocks and sections of such deeper nature, our remarks give some rather useful informations on them. Thus, for instance, the "maximality" feature of $\Pi$-blocks is given a formulation (Prop. 5 below) finer than the one given in [7]. Further, some new types of blocks and sections can be constructed, again in application of our remarks to such classical ones. These new blocks and sections give thus new orthogonality relations and we hope that some of them may turn to have some significance. There arize also several problems, which are stated at the end of the present note and to some of which we wish to come back elsewhere.

1. Orthogonality. Let $\&$ be a finite group, of order $g$, and $X$ be the set of all (absolutely irreducible ordinary) characters of $\$$. By a block equivalence in $\mathbb{B}$ we mean an equivalence relation $\mathfrak{B}$ of the set $X$, and each equivalence class in it we call a (B্-) block. With a subset $Y$ of $X, \mathfrak{B}(Y)$ denotes the join of the $\mathfrak{B}$-blocks containing an element of $Y$. If $\mathfrak{B}_{1}, \mathfrak{B}_{2}$ are two block equivalences, in $\mathfrak{B}$, the block equivalences $\mathfrak{B}_{1} \vee \mathfrak{B}_{2}, \mathfrak{B}_{1} \wedge \mathfrak{B}_{2}$ are defined in the sense of partial ordering of equivalence relations; each $\mathfrak{B}_{1} \vee \mathfrak{B}_{2}$-block is a non-

Received September 5, 1961. 
void intersection of a $\mathfrak{B}_{1}$-block and a $\mathfrak{B}_{2}$-block, while each $\mathfrak{B}_{1} \wedge \mathfrak{B}_{2}$-block is a minimal non-void subset of $X$ which is a join of $\mathfrak{B}_{i}$-blocks for each of $i=1,2$.

Let $R$ be the set of all conjugate classes in 6 . An equivalence relation $\subseteq$ of $\Omega$ is called a section equivalence and each equivalence class in it is called

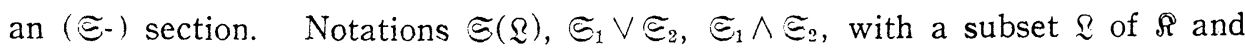

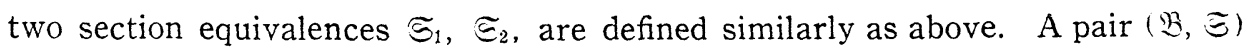
of a block equivalence and a section equivalence, in $\mathbb{B}$, is called a b.-s.-pair. For two such pairs $(\mathfrak{B}, \Xi)$, (B, $\Xi)$ we set

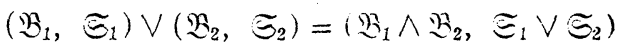

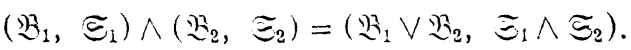

This introduces, as we easily verify, a lattice operation in the set of all b.-s. pairs.

Now, mere equivalences are naturally of little use by themselves, and our concern lies in their features as orthogonality and maximality. Thus, we say that the orthogonality of 1 st kind holds for a b.-s.-pair $(B, \Xi)$ when we have

$$
\sum_{x \in B} \%\langle K\rangle \bar{\gamma}\langle L\rangle=0
$$

for every $B$-block $B$ and any $K, L \in \Re$ with $\Xi(K) \oplus L$; here $\chi\langle K\rangle$ denotes the value $\chi(G)$ of $\%$ at any representative $G(\in \mathbb{B}$ ) of a class $K$ (and not the sum of values of $\%$ at all the elements of $K$ ). On the other hand, we say that the orthogonality of 2 nd kind holds for ( $(2, \Xi)$ ) when we have

$$
\sum_{K \in S} g_{K} \chi\langle K\rangle \bar{\Psi}\langle K\rangle=0
$$

for every $\Xi$-section $S$ and any $\%, \Psi \in X$ with $\mathcal{B}(\%) \nsubseteq \Psi$, where $g_{K}$ denotes the number of elements in $K$.

These two kinds of orthogonalities (for a fixed b.-s.-pair) turn out to be equivalent. So we say that the orthogonality holds for $(\mathfrak{B}, \subseteq)$ when either of the orthogonalities of 1 st and 2nd kinds holds, which thus implies the validity of both. Now we contend.

PRoposition 1. If the orthogonality holds for each of the b.-s.pairs ( $\mathbb{B}_{1}$, $\left.\Im_{1}\right),\left(\mathfrak{B}_{2}, \mathfrak{S}_{2}\right)$ in $\left(\mathfrak{B}\right.$, then it holds for $\left(\mathfrak{B}_{1}, \mathfrak{S}_{1}\right) \vee\left(\mathfrak{B}_{2}, \mathfrak{\subseteq}_{2}\right)$ and $\left(\mathfrak{B}_{1}, \mathfrak{S}_{1}\right) \wedge\left(\mathfrak{B}_{2}, \mathfrak{\Im}_{2}\right)$.

We prove this together with the above noted (and perhaps well known) equivalence of two kinds of partial orthogonalities, since such will clarify the 
logical structure of our proof (originating in the arguments of Brauer, Nagao, Osima and Tuan), not only that it is convenient. Thus we first prove

Lemma 1. Assume the validity of the orthogonality of 1 st kind for $(\mathbb{1}, \Xi)$. If a relation $\sum_{k \in t_{i}} a_{k} \%\langle K\rangle=0$ (with complex numbers $a_{k}$ ) holds for all $\%$ in a certain 3 -block $B$, then $\sum_{k \in S} a_{K} \%\langle K\rangle=0$ holds for every $\Xi$-section $S$ and every $\chi \in B$.

Proof. From the assumed relation we obtain

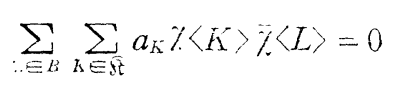

for every $L \in \Re$. Here we have $\sum_{x \subseteq B} \psi\langle K\rangle \bar{\psi}\langle L\rangle=0$ for every $K \notin \Xi\{L)$, by the orthogonality of 1 st kind for ( $\mathbb{E}, \Xi)$. It follows that

$$
\sum_{L \in \Xi} \sum_{h \in B} a_{K} \psi\langle K\rangle \bar{\psi}\langle L\rangle=0
$$

holds for every $L \in \hat{\Re}$. Multiplying $\bar{a}_{L}$ to this relation and taking the sum for $L \in S$, we obtain

$$
\sum_{L \in S} \sum_{K \in S} \sum_{x \in B} \bar{a}_{L} a_{K} \%\langle K\rangle \overline{\%}\langle L\rangle=\sum_{x \in B}\left|\sum_{K \in S} a_{K} \%\langle L\rangle\right|^{2}=0,
$$

and hence $\sum_{K \in S} a_{K} z\langle L\rangle=0(\mathcal{Y} \in B)$ as was asserted.

Lemma 2. Suppose that the orthogonality of lst kind holds for a b.-s.pair $\left(\mathrm{B}_{1}, \Xi_{1}\right)$ and the orthogonality of 2 nd kind holds for a second pair $\left(\mathfrak{B}_{2}, \Xi_{2}\right)$. Then the orthogonality of 2 nd kind holds for $\left(\mathcal{L}_{1}, \Xi_{1}\right) \vee\left(B_{2}, \Xi_{2}\right)$.

Proof. Let \%, $F$ be two characters of $\&$ such that $F=\mathbb{R}_{2}\left(\mathbb{B}_{1}(\%)\right)$, i.e. $\mathfrak{B}_{2}(\Psi) \cap \mathcal{B}_{1}(\%)=0$. By the orthogonality of 2 nd kind for $\left(\mathbb{N}_{2}, \Xi_{2}\right)$ we have $\sum_{h \in S_{2}} g_{K} \psi_{1}\langle K\rangle \bar{\Psi}\langle K\rangle=0$ for every character $\chi_{1}$ in $\mathbb{N}_{1}(\%)$, where $S_{2}$ is an arbitrary $\widetilde{\Xi}_{2}$-section. Since the orthogonality of 1 st kind holds for $\left(\mathbb{B}_{1}, \widetilde{S}_{1}\right)$, we then obtain $\sum_{K \in S_{1} \cap s_{2}} g_{K} \psi_{1}\langle K\rangle \bar{\Psi}\langle K\rangle=0$ for every $\Xi_{1}$-sectien $S_{1}$ and every $\%_{1} \in \mathbb{N}_{1}(\%)$. In particular

$$
\sum_{K \in S_{2} \cap S_{2}} g_{K} \%\langle K\rangle \bar{\Psi}\langle K\rangle=0
$$

(We can argue similarly in case $\Psi \notin \mathbb{R}_{1}\left(\mathcal{R}_{2}(\%)\right)$ is assumed. Indeed, this means $\% \notin \mathcal{L}_{2}\left(\mathcal{B}_{1}(\Psi)\right)$ and hence we may apply the above argument to $\Psi, \%$ in stead of $\%, F$, to obtain $\sum_{K \in s_{1} \cap s_{2}} g_{K} T\langle K\rangle \bar{\psi}\langle K\rangle=0$ which is equivalent to the above 
relation. Thus the same relation holds, in fact, whenever $\Psi \notin \mathfrak{B}_{1}\left(\mathfrak{B}_{2}(\chi)\right)$ $\cap \mathfrak{B}_{2}\left(\mathfrak{B}_{1}(\chi)\right)$.)

As $\mathfrak{B}_{2}\left(\mathfrak{B}_{1}(\mathcal{\chi})\right)$ is certainly contained in $\left(\mathfrak{B}_{2} \wedge \mathfrak{B}_{1}\right)(\chi)$, this proves our lemma. (Since $\mathfrak{B}_{2}\left(\mathfrak{B}_{1}(\chi)\right)$ is in general properly smaller than $\left(\mathfrak{B}_{2} \wedge \mathfrak{B}_{1}\right)(\chi)$, we see that our lemma is a very weak interpretation of a stronger fact. This becomes more definite when we observe the above remark in parentheses.)

Now, with an arbitrary b.-s.-pair $\left(\mathfrak{B}_{1}, \mathfrak{\subseteq}_{1}\right)$ we take as $\mathfrak{B}_{2}, \mathfrak{S}_{2}$ the $I$-(i.e. finest) equivalence and the 0 -(i.e. crudest) equivalence in $X, \AA$ respectively; the orthogonality of 2nd kind holds for this pair because of the ordinary orthogonality (while the orthogonality of 1st kind is trivial for it). It follows, by Lemma 2 , that the orthogonality of 1 st kind for $\left(\mathfrak{B}_{1}, \Im_{1}\right)$ entails the orthogonality of 2 nd kind for $\left(\mathfrak{B}_{1}, \mathfrak{S}_{1}\right) \vee(I, 0)=\left(\mathfrak{B}_{1}, \Im_{1}\right)$.

Quite similarly to Lemmas 1,2 we have

LEMMA 1'. Assume the validity of the orthogonality of 2 nd kind for (R, $\subseteq$ ). If a relation $\sum_{\chi \in X} a_{x} \chi\langle K\rangle=0$ (with complex numbers $a_{x}$ ) holds for all $K$ in $a$ certain $\subseteq$-section $S$, then $\sum_{x \in B} a_{x} \chi\langle K\rangle=0$ for every $B$-block $B$ and every $K$ in $S$.

LEMMA 2'. Suppose that the orthogonality of 2 nd kind holds for $\left(\mathfrak{B}_{1}, \bigodot_{1}\right)$ and the orthogonality of 1 st kind holds for $\left(\mathfrak{B}_{2}, \bigodot_{2}\right)$. Then the orthogonality of 1st kind holds for $\left(\mathfrak{B}_{1}, \widetilde{\subseteq}_{1}\right) \wedge\left(\mathfrak{B}_{2}, \Im_{2}\right)$.

Then on taking as $\mathfrak{B}_{2}, \Im_{2}$ this time the 0 - and $I$-equivalences, we see from Lemma $2^{\prime}$ (and the ordinary orthogonality relation) that the orthogonality of 2nd kind for a b.-s.-pair entails the orthogonality of 1st kind for the same pair. The equivalence of two partial orthogonalies (for a fixed pair) being thus shown, Lemmas $2,2^{\prime}$ imply evidently our Proposition 1.

Remark. Symmetry between blocks and sections would be made clearer if we should consider $\sqrt{g_{K} / g} \%\langle K\rangle$ in place of $\chi\langle K\rangle$.

2. Idempotents. To each $\% \in X$ there is associated a primitive idempotent $e_{x}=\frac{1}{g} \sum_{K \in \Omega} \chi(1) \bar{\gamma}\langle K\rangle K$ in the center 3 of the group algebra of $\mathbb{B}$ over the complexes $\Omega$, where the sum of the elements of a conjugate class $K$ is also denoted by $K$. To each $K \in \Re$ there is associated a primitive idempotent $\delta_{K}=\frac{g_{K}}{g} \sum_{\lambda \in X} \bar{\chi}\langle K\rangle \chi$ in the ring $\mathfrak{X}$ of class functions on $\mathcal{G}$ in $\Omega . \quad$ It is also well known that we have 


$$
\begin{aligned}
& K \boldsymbol{e}_{\chi}=\frac{g_{K} \chi\langle K\rangle}{g} \sum_{L \in \mathscr{\Re}} \bar{\chi}\langle L\rangle L, \\
& \chi_{\delta_{K}}=\frac{g_{K} \chi\langle K\rangle}{g} \sum_{\Psi \in X} \bar{\Psi}\langle K\rangle \Psi .
\end{aligned}
$$

Let $(\mathfrak{B}, \mathfrak{S})$ be a b.-s.-pair. For each $\mathfrak{B}$-block $B$ and for each $\mathfrak{S}$-section $S$ we set, after an idea originating from $\mathrm{H}$. Nagao and M. Osima [9],

$$
e_{B}=\sum_{\mathrm{X} \in B} e_{\mathrm{X}}, \quad \delta_{S}=\sum_{K \in S} \delta_{K} .
$$

The orthogonality of 1 st kind for $(\mathfrak{B}, \subseteq)$ means that for any $\mathfrak{B}$-block $B$ and S-section $S$ we have

$$
K \in S \Longrightarrow K e_{B} \in \sum_{L \in S} \Omega L
$$

Similarly the orthogonality of 2 nd kind is interpreted by

$$
\chi \in B \Rightarrow \chi \delta_{K} \in \sum_{\Psi \in B} \Omega \Psi
$$

If the orthogonality of 1 st kind holds for $\left(\mathfrak{B}_{1}, \mathfrak{C}_{1}\right),\left(\mathfrak{B}_{2}, \mathfrak{S}_{2}\right)$, then

$$
K e_{B_{1}} e_{B_{2}} \in\left(\sum_{L \in \mho_{1}(K)} \Omega L\right) e_{B_{2}} \subset \sum_{L \in \mathbb{S}_{2}\left(\mathbb{S}_{1}(K)\right)} \Omega L
$$

for any $\mathfrak{B}_{1}$-block $B_{1}$ and $\mathfrak{B}_{2}$-block $B_{2}$. Here $e_{B_{1}} e_{B_{2}}=\sum_{x \in B_{1} \cap B_{2}} e_{x}$ is the idempotent associated with the $\mathfrak{B}_{1} \vee \mathfrak{B}_{2}$-block $B_{1} \cap B_{2}$. (The equivalence of the orthogonalities of 1 st and 2 nd kinds being regarded as known) the above relation re-proves a one half of Proposition 1 . The other half is seen similarly by means of the idempotents $\delta_{K}$ in $\mathfrak{X}$.

3. Maximality. It is clear that if the orthogonality holds for a b.-s.-pair $(\mathfrak{B}, \mathfrak{S})$ and if $\left(\mathfrak{B}_{1}, \subseteq_{1}\right)$ is a second pair with $\mathfrak{B} \geq \mathfrak{B}_{1}, \subseteq \geq \bigodot_{1}$, then the orthogonality is valid for $\left(\mathfrak{B}_{1}, \Im_{1}\right)$ too.

Proposition 2. Given an equivalence $\subseteq$ in $\AA$, there is a unique finest equivalence $\mathfrak{B}$ in $X$ such that the orthogonality holds for $(\mathfrak{B}, \mathfrak{S})$.

Proof. Immediate from Prop. 1.

Note that we can give two explicit forms to Prop. 2 by taking the two kinds of orthogonality, 1 st and 2 nd. The equivalence $B$ in Prop. 2 is called the maximal block equivalence for $\subseteq$. Naturally a similar proposition holds with "block" and "section" interchanged, and for every equivalence in $X$ there is a 
unique maximal section equivalence for it.

Proposition 3. If for $i=1,2 \mathfrak{B}_{i}$ is maximal for $\dot{\Im}_{i}$ and if $\left(\mathfrak{B}_{3}, \varrho_{3}\right)$ $=\left(\mathfrak{B}_{1}, \Im_{1}\right) \vee\left(\mathfrak{B}_{2}, \mathfrak{\Im}_{2}\right)$, then $\mathfrak{B}_{3}$ is maximal for $\bigodot_{3}$.

Proof. Let $A$ be a subset of $X$ such that $\sum_{x \in A} \chi\langle K\rangle \bar{\chi}\langle L\rangle=0$ holds whenever $L \notin \bigodot_{3}(K)$. Since $L \notin \bigodot_{1}(K)$ naturally entails $L \notin \Im_{3}(K), \sum_{x \in A} \%\langle K\rangle \bar{\chi}\langle L\rangle$ must vanish then. As $\mathfrak{B}_{1}$ is maximal for $\varrho_{1}$, this implies that $A$ is a join of $\mathfrak{B}_{1}$ blocks. Similarly $A$ must be a join of $\mathfrak{B}_{2}$-blocks. So it is a join of $\mathfrak{B}_{3}$-blocks and this proves our proposition.

Remark. In Prop. 3 we can not, in general, replace $\vee$ with $\wedge$; see Example below.

Proposition 4. If a block equivalence $\mathfrak{B}$ is maximal for some section equivalence, there exists a unique finest section equivalence for which $B$ is maximal, and this section equivalence coincides with the maximal section equivalence for $B$.

Proof. This is clear from Prop. 3 (and an evident remark at the opening of this number).

4. Bearings on "classical" blocks. Except the consideration in 2, all above has little to do with the group structure of $\mathcal{S}$, and remains valid, with a suitable modification of terminology, whenever an "orthogonal" system of functions on a finite set is given (in place of group characters). It becomes significant only when it is combined with some group properties of $\mathbb{B}$, directly or indirectly. So we want to consider its bearings on the "classical" blocks and sections defined arithmetico-group-theoretically. Thus, fundamental are Brauer's block and section equivalences $\mathfrak{B}_{p}$, $\bigodot_{p}$ defined with respect to each prime $p$ (dividing $g$ ); the orthogonality of 1 st kind for $\left(\mathfrak{B}_{p}, \Im_{p}\right)$ was proved by Brauer by means of his fundamental theorem on decomposition numbers (Brauer [1]; cf. Iizuka [6]). and the orthogonality of 2nd kind was derived from it by himself and Osima [10]. The pair $\left(\mathfrak{B}^{p}, \mathfrak{\subseteq}^{p}\right)$ of $p$-complementary block and $p$ regular section equivalences, studied in Iizuka [5], is in a sense dual to $\left(B_{p}\right.$, $\left.\Xi_{p}\right) ; p$-regular sections were first called "p-Oberklassen" by Roquette [11]. Iizuka [7], one of the present writers, further studied more general notions of $\Pi$-blocks and $\Pi$-sections associated with every finite set $\Pi$ of primes (dividing $g) ; \mathfrak{B}_{\mathrm{n}}=\wedge \mathfrak{B}_{p}, p$ running over primes (dividing $g$ and) belonging to $I I$ and 
$\varsigma_{n}=\wedge \varsigma^{q}, q$ running over primes (dividing $g$ and) not belonging to $I I$.

Now, $\mathfrak{B}_{p}$ is maximal for $\mathfrak{S}_{p}$; this was proved in Osima [9], Theorem 3 and can be derived readily from Brauer-Feit [2] too. From our Prop. 3 follows

PROPOSITION 5. (II-block equivalence) $B_{n}$ is maximal for $\bigodot_{p_{1}} \vee \cdots \vee \bigodot_{p_{t}}$ $\left(\Pi=\left\{p_{1}, \ldots, p_{t}\right\}\right)$.

We are reporting this particularly because this is finer than [2.3. C] in [7] (where the maximality of $\mathfrak{B}_{n}$ for $\mathfrak{\subseteq}_{n}$ is asserted). Indeed, the conjugate classes of two elements $G, H$ of $\mathscr{S}$ are equivalent by $\aleph_{n}$ if and only if the $\Pi$-factors $G_{\mathrm{n}}, H_{\mathrm{n}}$ of $G, H$ are conjugate (see [2.4 A] in [7]), while they are equivalent by $\Im_{p_{1}} \vee \ldots \vee \Im_{p_{t}}$ if and only if for each $i=1, \ldots, t$ the $p_{i}$-factors $G_{p_{i}}, H_{p_{i}}$ are conjugate. So $\varsigma_{n}$ is properly finer than $\varsigma_{p_{1}} \vee \cdots \vee \varsigma_{p_{l}}$ in general, as an easy example shows.

On the other hand, the orthogonality of $\left(\mathcal{B}_{\mathrm{n}}, \Im_{p_{1}} \vee \cdots \vee \Im_{p_{t}}\right)$, which follows from the orthogonality of $\left(\mathfrak{B}_{p_{i}}, \Im_{p_{i}}\right), i=1, \ldots, t$, and our (elementary and rather formal) Prop. 1 , is weaker than the orthogonality of $\left(\mathfrak{B}_{n}, \mathfrak{S}_{n}\right)$ proved in lizuka [7] by combining the cases of $\left(\mathfrak{B}_{p_{i}}, \Im_{p_{i}}\right)$ arithmetically and group-theoretically, so to speak.

That $\mathfrak{S}_{\mathrm{n}}$ is in general not maximal for $\mathfrak{B}_{\mathrm{n}}$ (and indeed $\mathfrak{S}_{p}$ (resp. $\mathfrak{S}^{p}$ ) is in general not maximal for $\mathfrak{B}_{p}\left(\right.$ resp. $\left.\mathfrak{B}^{p}\right)$ ) was noted in [7]. An example for this is given e.g. by $\mathfrak{B}_{2}, \varrho_{2}$ in the symmetric group $S_{4}$. Symmetric groups provide also instances for our remark at the end of the preceding number. Thus

Example. Let $\mathbb{B}$ be the symmetric group $S_{30}$. Then 8 characters associated with Young's diagrams

$$
1^{30},(30)^{1}, 2^{15},(15)^{2}, 3^{10},(10)^{3}, 5^{6}, 6^{5}
$$

are all equivalent in each of $\mathfrak{B}_{2}, \mathfrak{B}_{3}, \mathfrak{B}_{5}$ (see [3]). This shows that $\mathfrak{B}_{2} \vee \mathfrak{B}_{3} \vee \mathfrak{B}_{5}$ is not the identity equivalence $I$. On the other hand, $\Xi_{2} \wedge \Im_{3} \wedge \varsigma_{5}$ is the 0 equivalence, as Prop. 6 below shows, and $\mathfrak{2}_{2} \vee \mathfrak{B}_{3} \vee \mathfrak{B}_{5}$ is certainly not maximal for $\Im_{2} \wedge \Im_{3} \wedge \Im_{5}$, showing the validity of our cited Remark.

PRoposition 6. Suppose the order $g$ of $\mathbb{B}$ is divisible by at least 3 distinct primes $p, q, r$. Then $\Im_{p} \wedge \subseteq_{q} \wedge \Im_{r}$ is the 0-equivalence.

Proof. Let $G, H$ be two arbitrary elements of $\$$. Let $P$ be the $p$-factor 
of $G$, and let $P_{1}, R_{1}$ be respectively the $p$ - and the $r$-factors of $H$. Then the class $[P]$ of $P$ is $\Im_{p}$-equivalent to the class $[G]$ of $G$, i.e. $[P] \in \Im_{p}([G])$, and $\left[P_{1} R_{1}\right] \in \Im_{g}([P])=\Im_{q}\left(\Im_{p}([G])\right)$. Further $[H] \in \Im_{r}\left(\left[P_{1} R_{1}\right]\right)$. Hence $[H]$ $\in \mathfrak{S}_{r}\left(\Im_{q}\left(\Im_{p}([G])\right)\right)$, proving our proposition.

We remark that in the above example we have seen that $\mathfrak{B}_{p} \wedge \mathfrak{B}_{q} \wedge \mathfrak{B}_{r}$, with distinct primes $p, q, r$, is not trivial in general.

5. Subgroups. Besides ( $p$-, $p$-complementary and) $I I$-blocks, also bocks with respect to normal subgroups, which generalize Osima's [8] blocks, have been studied ([7]). However, without making studies of the bearings of our general observations on those blocks with respect to normal subgroups, similar as above, we turn to proving a certain relation beetween the orthogonality and a subgroup. Thus

Proposition 7. Let 5 be a subgroup of $\mathbb{S}$ and $h$ be its order. If $(\mathfrak{B}, \mathbb{S})$ is $a$ b.-s.-pair in $\mathbb{B}$ satisfying the orthogonality, if $B, S$ are any $\mathfrak{B}$-block and $\mathbb{S}$ section, then

$$
\sum_{L \in S} h(L) \sum_{\chi \in B} \chi\langle K\rangle \bar{\chi}\langle L\rangle \equiv 0 \quad \bmod h
$$

(in the domain of algebraic integers) for every $K \in S$, where $h(L)$ denotes the number of elements in $\$ 2$ belonging to a conjugate class $L$ in $\mathbb{B}$.

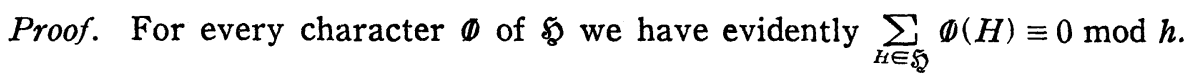
The same holds when $\Phi$ is a linear combination of characters of $\mathfrak{g}$ with algebraic integers as coefficients. We set in particular $\emptyset(H)=\sum_{\chi \in B} \chi\langle K\rangle \bar{\gamma}_{(}(H)$, and observe that $\Phi(H)$ vanishes whenever the conjugate class in $\$ B$ of $H$ does not belong to $S$. Thus the congruence $\sum_{h \in \mathfrak{S}} \emptyset(H) \equiv 0(\bmod h)$ becomes the one in our proposition.

This proposition originates from Brauer-Tuan [4], Lemma 3, and contains it as a special case where $g$ is divisible by two distinct primes $p$ and $q$, $\&$ has no element of order $p q, K$ is the class of a $p$-singular element, and a $q$-Sylow group is taken for $\$$

6. Remarks. As the $\Pi$-section equivalence $\varsigma_{\mathrm{n}}$ is not, in general, maximal for $\mathfrak{B}_{\mathrm{n}}$, it is natural to look for the unique section equivalence, in $\mathbb{R}$, maximal for $\mathfrak{B}_{\mathrm{n}}$ and to study if it can directly be characterized arithmetically and grouptheoretically; cf. Prop. 4 for a two-fold character of this section equivalenc. 
Special cases of this problem concern naturally with $\mathfrak{B}_{p}$ and $\mathfrak{B}^{p}$.

Symmetric groups were seen above to provide some counterexamples. The other extreme types of groups, i.e. abelian and nilpotent groups are quite tame. It may be of interest to determine all types of groups which behave tamely, i.e. groups in which e.g. $\mathfrak{S}_{11}\left(\right.$ or $\mathfrak{\subseteq}_{p}$, or $\mathfrak{S}^{p}$ ) is always maximal for $\mathfrak{B}_{\mathrm{n}}$ (or $\mathfrak{b}_{p}$, or $\mathfrak{B}^{p}$, respectively).

On the other hand, it seems to us also of interest to determine all block equivalences whatsoever which are maximal with respect to some section equivalences. Their totality is, by Prop. 3, closed under forming $\wedge$.

By means of our operations $\wedge$ and $\vee$ we can construct new b.-s.pairs, satisfying the orthogonality, starting from those of II-blocks and II-sections with varying $\Pi$ (or those of blocks and sections (called subsections in [7]) with respect to normal subgroups). Though Prop. 6 indicates that many of such new b.-s.-pairs may turn to be trivial ones and though our comparison of $\left(\mathfrak{B}_{p_{1}}\right.$, $\left.\subseteq_{p_{1}}\right) \vee \cdots \vee\left(B_{p_{1}}, \subseteq_{p_{1}}\right)$ and $\left(\mathfrak{B}_{n}, \subseteq_{n}\right)$, made after Prop. 5 , shows that many of such orthogonality relations may be rather weak ones, the construction of such new b,-s.-pairs will, as it seems to us, be useful for clarifying the nature of orthogonality relations in finite groups, beside their material bearings as shown in Prop. 5 and their actual applications as illustrated in [4].

As is seen in [7] $\Pi$-blocks and $\Pi$-sections are closely connected with the arithmetic of the center of the group ring and the ring of class functions and can indeed be characterized by primitive idempotents in some subrings of those arizing from rational arithmetic. It is perhaps of use to consider the significance the new b.-s.-pairs as above will have to subrings of more general types.

Another problem in this context will be to look for characteristic features of characters with respect to the orthogonalities, by considering "orthogonal" systems of functions on the set $\mathfrak{K}$ of conjugate classes other than that of characters.

\section{REFERENCES}

[1] R. Brauer, Zur Darstellungstheorie der Gruppen endlicher Ordnung, II, Math. Zeits. 72 (1959), 25-46.

[2] R. Brauer and W. Feit, On the number of irreducible characters of finite groups in a given block, Proc. Nat. Acad. Sci. USA 45 (1959), 361-365.

[ 3 ] R. Brauer-G. de B. Robinson, On a conjecture by Nakayama, Trans. Roy. Soc. Canada 3. S. 41 (1947), Sec. III, 11. 
[4] R. Brauer-H. F. Tuan, On simple groups of finite order, I, Bull. Amer. Math. Soc. 51 (1945), 756-766.

[5] K. Iizuka, On the blocks and the sections of finite groups, Kumamoto J. of Sci. 5 (1960), 53-62.

[6] - On Brauer's theorem on sections in the theory of blocks of group characters, Math. Zeits. 75 (1961), 299-304.

[7] - Some studies on the orthogonality relations for group characters, Kumamoto J. of Sci. 5 (1960), 111-118.

[ 8 ] M. Osima, On the representations of groups of finite order, Math. J. Okayama Univ. 1 (1.952), 33-61.

[ 9 ] - - Notes on blocks of group characters, Math. J. Okayama Univ. 4 (1955), 175-188.

[10] - On some properties of group characters, Proc. Japan Acad. 36 (1960), 18-21.

[11] P. Roquette, Arithmetische Untersuchung des Charakterringes einer endlichen Gruppe, Jour. f.d.r.u.a. Math. 190 (1952), 148-168.

Tôhoku University and Kumamoto University

Nagoya University 